\title{
Estimación sobre niveles y factores de riesgo psicosocial en adolescentes escolarizados de Ambato, Ecuador
}

\author{
Rodrigo Moreta ${ }^{\star}$, Carlos Reyes ${ }^{\star *}$, Marlon Mayorga ${ }^{\star * *}$, Lorena León-Tamayo ${ }^{\star * * *}$
}

\footnotetext{
Doctor en Psicología Clínica y de la Salud. Profesor-investigador titular, Escuela de Psicología, Pontificia Universidad Católica del Ecuador, Ecuador. Correo electrónico: rmoreta@pucesa.edu.ec

* Doctor en Comportamiento Social y Organizacional. Profesor-investigador, Facultad de Psicología, Universidad Politécnica Salesiana del Ecuador, Ecuador. Correo electrónico: creyes@ups. edu.ec

*** Doctor en Psicología Clínica. Profesorinvestigador, Facultad de Psicología, Universidad Politécnica Salesiana del Ecuador, Ecuador. Correo electrónico: pmayorga@pucesa.edu.ec

${ }_{* * * *}$ Magíster en Psicoanálisis con mención en educación. Profesora-investigadora titular, Facultad de Psicología, Pontificia Universidad Católica del Ecuador, sede Ambato, Ecuador. Correo electrónico: aleon@pucesa.edu.ec
}

Recibido: 10 de noviembre del 2016 Aprobado: 11 de julio del 2017

Cómo citar este artículo: Moreta, R., Reyes, C., Mayorga, M. y León-Tamayo, L. (2017). Estimación sobre niveles y factores de riesgo psicosocial en adolescentes escolarizados de Ambato, Ecuador. Pensando Psicología, 13(22), 29-40. doi: https://doi.org/10.16925/ pe.v13i22.1986

\section{Resumen}

Objetivo: determinar los niveles de riesgo psicosocial en adolescentes escolarizados de la ciudad de Ambato, Ecuador. Método: se trabajó con un grupo de 302 adolescentes con edades comprendidas entre 14 y 18 años de cuatro instituciones educativas. La evaluación se realizó a través del Inventario Autodescriptivo del Adolescente (IADA). Resultados: los resultados mostraron que los niveles de práctica de comportamientos y situaciones de riesgo se encuentran por debajo del punto de corte en todos los niveles evaluados. Los hombres presentan mayores diferencias $(t(203)=3,094, p<0,001)$ que las mujeres en la dimensión de riesgos a la salud. También se identificó un subgrupo vulnerable del $20 \%$ de los participantes en la evaluación del riesgo en el área escolar y otro subgrupo vulnerable del $17 \%$ en mujeres en la dimensión familia. Conclusiones: la existencia de antecedentes psicológicos familiares e individuales constituyen factores de riesgo que están presentes en más comportamientos y situaciones de riesgo. Se discuten los alcances de estos resultados en futuros planes de prevención e intervención con población adolescente ecuatoriana.

Palabras clave: adolescentes, escolarización, planes de prevención e intervención, situaciones de riesgo. 


\title{
Estimating Psychosocial Risk Factors and Levels in Adolescents Attending School in Ambato, Ecuador
}

\begin{abstract}
Purpose: To determine the psychosocial risk levels in adolescents attending school in Ambato, Ecuador. Method: We worked with a group of 302 adolescents between 14-18 years old, from four educational institutions. The assessment was made through the Self-Description Inventory (SDI) for adolescents. Results: The results showed that the levels of risk behaviors and situations are below the cut-off point at all levels evaluated. Men exhibited greater differences $(t(203)=3.094$, $\mathrm{p}<0.001)$ than women in the health risk dimension. We identified two vulnerable subgroups in the risk assessment: $20 \%$ of participants in the school dimension and $17 \%$ of women in the family dimension. Conclusions: Family and individual psychological history constitutes a risk factor that is present in more risk behaviors and situations. The scope of these results in future prevention and intervention plans with Ecuadorian adolescents is discussed.
\end{abstract}

Keywords: adolescents, schooling, prevention and intervention plans, risk situations.

\section{Estimativa sobre níveis e fatores de risco psicossocial em adolescentes escolarizados de Ambato, Equador}

\section{Resumo}

Objetivo: determinar os níveis de risco psicossocial em adolescentes escolarizados da cidade de Ambato, Equador. Método: trabalhou-se com um grupo de 302 adolescentes com idades compreendidas entre 14 a 18 anos, de quatro instituições educativas. A avaliação foi realizada por meio do Inventário Autodescritivo do Adolescente (Iada). Resultados: os resultados mostraram que os níveis de prática de comportamentos e situações de risco se encontram abaixo do ponto de corte em todos os níveis avaliados. Os homens apresentam maiores diferenças $(\mathrm{t}(203)=3,094, \mathrm{p}<0,001)$ do que as mulheres na dimensão de riscos à saúde. Também foi identificado um subgrupo vulnerável de $20 \%$ dos participantes na avaliação do risco na área escolar e outro subgrupo vulnerável de $17 \%$ em mulheres na dimensão família. Conclusões: a existência de antecedentes psicológicos familiares e individuais constitui fatores de risco que estão presentes em mais comportamentos e situações de risco. Discutem-se os alcances desses resultados em futuros planos de prevenção e intervenção com população adolescente equatoriana.

Palavras-chave: adolescentes, escolarização, planos de prevenção e intervenção, situações de risco. 


\section{Introducción}

Los comportamientos de riesgo forman parte de los estilos de vida que se presentan en un contexto (Toro-Trallero, 2010). Un "riesgo" se entiende como la probabilidad de que se produzca un evento que genere potenciales consecuencias negativas (United Nations International Strategy for Disaster Reduction [UNISDR], 2009). Se ha identificado que los niveles implicados o afectados por el riesgo pueden ser: físicos, sociales, económicos o psicológicos. La presencia de comportamientos de riesgo en adolescentes, y especialmente su progresión, deben ser tomados como un indicador al cual se debe responder con acciones concretas y viables (Páramo, 2011).

Por lo expuesto, el objetivo del presente estudio consiste en determinar los niveles de riesgo en adolescentes escolarizados de la ciudad de Ambato, Ecuador. Además, de manera complementaria, se busca identificar la proporción de participantes con criterios de vulnerabilidad. Dichos criterios se basan en la probabilidad de que constituyan o no factores de riesgo y su estimación requiere abordar características como el sexo, la edad, la identificación étnica, el domicilio, el centro de estudios o los antecedentes psicológicos, entre otras.

\section{Situación de los adolescentes escolarizados en Ecuador y en la ciudad de Ambato}

En Ecuador, el promedio de años de escolaridad para adolescentes es de 9,7 años, siendo este promedio más alto en el área urbana (11 años), que en la rural (6,9 años). En cuanto al sexo, el tiempo de escolaridad de los hombres es de 9,9 años y el de las mujeres es de 9,6 años. En cuanto a la distribución por etnias, la media de escolaridad corresponde a 5,9 años en población indígena, a 10,1 años para mestizos, a 8,9 años para el grupo de afroecuatorianos y a 6,7 años en montubios (Instituto Nacional de Estadística y Censos [INEC], 2010).

En los adolescentes, las cifras en relación con la asistencia a un establecimiento educativo tienen una tendencia a la baja a medida que cumplen más edad: asiste el $89,7 \%$ de jóvenes de 15 años, el $86,1 \%$ de aquellos de 16 años y el 77,4\% de quienes tienen 17 años. Con respecto a cursar bachillerato, durante el periodo 2012-2013 se identificaron en Ecuador 708239 estudiantes, con una cifra prácticamente idéntica entre mujeres $(50,9 \%)$ y hombres $(49,1 \%)$ (INEC, 2010).
En cuanto al lugar de procedencia, los estudiantes del sector urbano corresponden al $96,5 \%$ y los del sector rural, al 83,3\%; siendo el 93,7\% población masculina y el $89,6 \%$ población femenina. Según el nivel educativo y el tipo de establecimiento, el 69,3\% de estudiantes está inserto en instituciones públicas, el 6,5\% en instituciones fiscomisionales, el 22,8\% en privadas y el $1,4 \%$ en municipales (Ministerio de Educación de Ecuador, 2014a y 2014b).

En la ciudad de Ambato, el total de estudiantes de bachillerato registrados en el periodo 2012-2013 fue de alrededor de 17607 estudiantes, distribuidos de manera idéntica a la tasa nacional en el 50,9\% de mujeres y el $49,1 \%$ de hombres. Provienen en su mayoría del sector urbano $(77,8 \%)$, con una menor presencia del área rural $(22,2 \%)$. Según el tipo de institución, el $75 \%$ procede de instituciones fiscales; el 3,6\%, de fiscomisionales; y el $21,4 \%$, de centros privados. La distribución de los estudiantes según los años de bachillerato es: 38,1\% estudia en el primer año de bachillerato; $31,4 \%$, en el segundo año; y 31,5\%, en el tercer año (Ministerio de Educación de Ecuador, 2014c).

\section{Riesgo y comportamientos de riesgo}

El riesgo comúnmente se asocia a la presencia de factores que resaltan o inhiben la eventualidad de enfermedad o déficit y a los respectivos procesos subyacentes (Irwin, 1990; Rutter, 1987) que afectan la cotidianidad y la seguridad de las personas ante tales eventos (Cardona, 2001). Estos factores influyen en la posible aparición de dificultades, enfermedades, déficits, trastornos u otros similares. Son identificados como "factores o indicadores de riesgo" los elementos que se desprenden de cualquier tipo de circunstancia o evento de naturaleza biológica, psicológica, social o medioambiental, cuya presencia o ausencia influye en la posibilidad de que se presente un problema en el desarrollo de un individuo o un grupo.

Se ha establecido que los factores o indicadores de riesgo están compuestos por dos elementos: la "amenaza" y la "vulnerabilidad" (UNISDR, 2009). La amenaza corresponde a un fenómeno, sustancia, actividad humana o condición peligrosa que puede ocasionar la muerte, lesiones u otros impactos en la salud. En adolescentes, constituyen amenazas los aspectos disfuncionales en el ambiente familiar, escolar o social, y el uso de sustancias, entre otros (Becoña, 2002). La literatura identifica que la adolescencia representaría una etapa de desarrollo amenazante cuando le impone al joven exigencias que no está preparado para resolver, pues carece de la madurez física 
y psíquica suficiente (Coleman, Hendry y Kloeb, 2007). De allí que la probabilidad de ocurrencia de una conducta de riesgo sea mayor en esta etapa, dado que el adolescente puede adoptar comportamientos de autoafirmación personal que incrementan su vulnerabilidad (Arnett, 1992 y 1999). Por otro lado, se ha observado que un evento representa una amenaza cuando se presentan dos parámetros cuantitativos asociados a esta: la intensidad y la frecuencia (UNISDR, 2009). Así, la presencia única y esporádica de una situación considerada como amenaza no configura un riesgo como tal.

El segundo componente, la vulnerabilidad, se refiere a la capacidad disminuida de una persona para anticiparse, confrontar y tolerar los efectos de un daño para su posterior recuperación (International Federation of Red Cross [IFRC], 2014; SánchezGonzález y Egea-Jiménez, 2011). Se han identificado factores internos y externos que promueven mayor vulnerabilidad; entre los primeros están la baja autoestima, la baja autoeficacia o la depresión (Bardagi, Arteche y Neiva-Silva, 2005), mientras que entre los segundos se hallan características relacionadas con aspectos sociodemográficos (Cepal, 2001). Las condiciones socioeconómicas precarias influirían en las conductas de desadaptación social y en la presencia de síntomas psicológicos (Cardozo y Alderete, 2009). De esta manera, la vulnerabilidad estaría expresada en la exposición que sitúa al individuo en una posición de desventaja ante una situación de riesgo (Cardona, 2001), lo cual podría desencadenar la aparición de problemas o su agravamiento.

Un término derivado de riesgo es "situación de riesgo", que se refiere a la circunstancia en la que el individuo realiza el intento por frenar una conducta de manera inadecuada (Witkiewitz y Marlatt, 2004). Concretamente, corresponde a los intentos por detener, con los recursos del individuo, los efectos del riesgo para aplacar las consecuencias próximas del riesgo. No obstante, estos intentos no producen resultados positivos, con lo cual se podrían incrementar o maximizar dichos efectos de riesgo.

\section{Riesgos psicosociales y comportamientos de riesgo en el adolescente}

Según Toro-Trallero (2010), el comportamiento de riesgo en adolescentes se define como una serie de conductas que proveen gratificaciones moderadas o importantes a corto plazo y potenciales perjuicios a largo plazo. Estos comportamientos en adolescentes pueden ser identificados de acuerdo con los siguientes indicadores de riesgo psicosociales:

1. Insatisfacción de necesidades psicológicas básicas (Bozhovich, 1976). Las necesidades psicológicas que están ligadas al desarrollo durante la adolescencia son variables y al manifestarse, se convierten en necesidades a ser satisfechas. Aquí se incluye el deseo de explorar y de adquirir experiencias. Es necesario considerar cómo los riesgos y los comportamientos de riesgo relacionados con estas necesidades tienen repercusión en la psicopatología juvenil, especialmente en factores de riesgo relacionados con la autoestima o la autoimagen (Haquin, Larraguibel y Cabezas, 2004).

2. Patrones no adecuados en torno a la crianza y la educación. Incluyen la sobreprotección, el autoritarismo y la agresión física o verbal, asociado a la dificultad en la adopción adecuada de normas de conducta y disciplina, lo cual puede producir un efecto de frustración entre los familiares encargados de la crianza (Herrera, 1999). También, hacen referencia a la falta o al incumplimiento de reglas debido a problemas en el manejo de los roles familiares y sociales, lo que dificulta el sano y libre desarrollo de la personalidad del adolescente (Arce, 1995). Por el contrario, un adecuado vínculo familiar con el joven ayuda a disminuir significativamente la probabilidad de la manifestación de conductas de riesgo (Friedman, 2006; HuitrónBravo et al., 2011).

3. Ambiente frustrante. Corresponde a la ausencia o déficit de manifestaciones de afecto, a la intromisión en la vida privada, a la censura inmotivada y a las amenazas y castigos en el adolescente (Bozhovich, 1976; Herrera, 1999). Estos factores inciden en el aparecimiento de trastornos afectivos, depresión, autolesiones o intentos autolíticos (Fergusson, Woodward y Horwood, 2000), los cuales también pueden incluir conductas relacionadas con desórdenes alimenticios (Fisher y Smith, 2008) o con intentos del adolescente por controlar su cuerpo. Un estudio en México identificó que los indicadores de riesgo en este nivel se vinculan a la interacción con los padres, la autoimagen y el consumo precoz de tabaco y alcohol, siendo mayor en adolescentes hombres que en mujeres (Barcelata, Durán y Lucio, 2004).

4. Sexualidad mal orientada. Se refiere a la ausencia o bloqueo en la familia de la información adecuada 
en relación con la sexualidad y a la búsqueda del adolescente de dicha información. El despertar sexual, la curiosidad y el deseo de experimentar representan conductas naturales y esperadas en el adolescente, pero se vuelven conductas de riesgo cuando una inadecuada orientación interrumpe esta búsqueda (Sinha, 2007). Los mitos en torno al sexo incrementan esta posibilidad, que se expresa en embarazos no planeados, abortos y/o enfermedades de transmisión sexual (Vargas-Trujillo, Henao y González, 2007). El sexo masculino o femenino se contempla como una variable que incide en el incremento del riesgo, siendo mayor para los varones (Campo-Arias, Cogollo y Díaz, 2008). También, se observa que los adolescentes que perciben a sus familias como disfuncionales tienden a iniciar relaciones sexuales más temprano de lo esperado (Huitrón-Bravo et al., 2011).

5. Pertenencia a grupos antisociales. Surge como consecuencia de la necesidad de autoafirmación individual y de la ausencia de un entorno de desarrollo. Esto puede conducir al adolescente a la búsqueda de la aceptación y afirmación grupal y a la fácil asimilación de conductas no aceptadas socialmente (Herrera, 1999). La vulnerabilidad socioeconómica del adolescente y de la familia es un factor asociado a estas prácticas. En las conductas antisociales, se evidencia una correlación con los conflictos familiares, los problemas de comunicación y el estilo educativo parental (Villar-Torres et al., 2003).

6. Escasas oportunidades escolares, culturales y económicas. Se refiere a la ausencia o déficit de disponer de condiciones u oportunidades para el desarrollo, el crecimiento o la posibilidad de potenciar habilidades y capacidades en los adolescentes. Se identifican como la principal fuente de riesgo sociocultural (Mattar, 2001) y, en consecuencia, dificultan las posibilidades de desarrollo personal de los adolescentes para asumir responsabilidades y afrontar problemas (Larson, 1988). Aquí se incluyen conductas como: inicio precoz en actividades laborales, bajo rendimiento escolar, problemas de aprendizaje o deserción escolar. Por ejemplo, en la Ciudad de México, en un estudio realizado con 1.000 jóvenes entre 12 y 22 años, se encontró que estas conductas de riesgo están presentes en aquellos que presentan bajo desempeño escolar. Paralelamente, se ha encontrado una asociación entre diversas conductas de riesgo y la interacción de los padres y la presión social de los pares adolescentes (Palacios y Andrade, 2007).
La situación de los adolescentes en Ecuador, en particular en la ciudad de Ambato, constituye una temática poco explorada. Se asume la presencia de cambios significativos en varios ámbitos, tales como el grupo familiar, el entorno social y escolar, el desarrollo personal y la salud física y mental. Por otro lado, surgen interrogantes en relación con la presencia de ciertos riesgos que puedan expresarse en el origen de conductas de riesgo. No obstante, en Ecuador no hay estudios que pongan de manifiesto dichos comportamientos.

A partir de estos antecedentes, en el presente estudio se explorarán los niveles de comportamiento y situaciones de riesgo en adolescentes de Ambato, observando las diferencias por sexo, edad, área de procedencia, institución educativa, identificación étnica y antecedentes psicológicos. Dado que no hay estudios previos, no se establecerán hipótesis en este punto. El objetivo es obtener un perfil de riesgo psicosocial de los adolescentes, identificando qué factores se asocian a tal riesgo, y confirmar la fiabilidad de las puntuaciones de los ítems de la prueba utilizada.

\section{Método}

\section{Participantes}

Participaron 302 adolescentes escolarizados de la ciudad de Ambato. El grupo estuvo conformado por 225 hombres $(77,2 \%)$ y 66 mujeres $(22,8 \%)$, con edades comprendidas entre 14 y 18 años $(M=15,6$ años, $D S=1,0)$. La distribución por edad comprende el 8,3\% con 14 años, el $44,4 \%$ con 15 años, el 26,5\% con 16 años, el $16,2 \%$ con 17 años y el 4,6\% con 18 años. Respecto a la identificación étnica, el $84,4 \%$ se identificó como mestizo, el 7,3\% como blanco, el $5,6 \%$ como indígena y el $2 \%$ como afroecuatoriano. El $66,7 \%$ proviene del sector urbano y el $33,3 \%$ proviene del sector rural de la ciudad. En referencia a las características familiares, el 39,5\% de los participantes corresponde a hijos mayores, el 27,8\% a hermanos intermedios y el $24,7 \%$ a hijos menores. El 7,9\% corresponde a hijos únicos.

En relación con los años de bachillerato cursados, el 63,9\% de los participantes cursa primer año de bachillerato, el 17,5\% cursa segundo año y el $18,6 \%$ cursa tercer año. Además, el $43 \%$ asiste a centros educativos públicos (unidades educativas Luís A. Martínez, Las Américas y Joaquín Lalama) y el $57 \%$ acude a un centro educativo religioso-privado (Colegio Diocesano Pío x). Por último, el 11,5\% de 
los participantes reportó antecedentes psicológicos individuales y el $6,2 \%$ cuenta con antecedentes psicológicos familiares.

El método de muestreo utilizado corresponde al de tipo no probabilístico, y los criterios de inclusión para la selección de los participantes fueron: a) voluntad para participar en la evaluación; b) contar con el permiso firmado del representante legal o tutor; c) ser un estudiante de asistencia regular a clase; d) ser estudiante domiciliado en la ciudad de Ambato; e) tener una edad comprendida entre los 14 y los 18 años; y f) cursar entre el primer y tercer año de bachillerato.

\section{Medidas}

Inventario Autodescriptivo del Adolescente (IADA). Según Gómez-Maqueo, Barcelata y Durán (2010), este instrumento consta de 162 ítems para participantes con un nivel de estudios secundarios y de 165 ítems para el nivel bachillerato. Esta versión cuenta con 33 ítems descriptivos y 134 ítems que evalúan el riesgo en cinco áreas de la vida de los adolescentes: a) familia con 48 ítems (entre 0 y 275 puntos), social con ocho ítems (entre 0 y 75 puntos), personal con 15 ítems (entre 0 y 175 puntos), escolar con 28 ítems (entre 0 y 125 puntos) y salud con 33 ítems (entre 0 y 185 puntos). Los puntajes altos evidencian la presencia de mayor riesgo psicosocial. La escala de respuesta del instrumento es dicotómica (sí y No). El criterio de calificación para considerar la presencia significativa de riesgo corresponde a: familia $=$ mayor a 155 puntos; social $=$ mayor a 57 puntos; personal $=$ mayor a 122 puntos; escolar = mayor a 83 puntos; $y$ salud $=$ mayor a 135 puntos. Todas estas puntuaciones referenciales se encuentran ocupando la puntuación típica 80 $\left(T_{(80)}\right)$ en las diferentes dimensiones.

En relación con las propiedades psicométricas del IADA, presenta una consistencia interna ( $\alpha$ ) en cada una de las dimensiones de entre 0,63 y 0,83 , y en el análisis global, de 0,90 . Estos datos se obtuvieron tras un estudio de 1.660 adolescentes de 13 a 18 años de edad procedentes de centros educativos de la Ciudad de México. La validez discriminante muestra además una capacidad para diferenciar adolescentes que presenten características normales y características clínicas $t(302)=-2,22 ; p<0,05$. En la identificación de la estabilidad temporal del IADA, la prueba test-retest $(r)$ revela valores fluctuantes entre 0,29 y 0,91 . Respecto a la validez de constructo, la versión del IADA para bachilleres muestra una estructura del test compuesta por las cinco dimensiones enunciadas antes. Las correlaciones ( $r$ ) entre los puntajes de cada una de las dimensiones con el puntaje global del IADA oscilan entre 0,41 (Social) y 0,84 (Familia).

\section{Procedimiento y análisis de datos}

Se estableció contacto con el personal administrativo y directivo de los centros educativos públicos y privados de la ciudad de Ambato, donde se entregó información acerca de los objetivos del estudio y se solicitó la autorización para la participación de los estudiantes. Se plantearon como criterios de inclusión a estudiantes que estuvieran cursando el año escolar, que tuvieran como edad mínima 15 años y no padecieran trastornos mentales. Luego, se realizó el contacto inicial con los estudiantes, en el que se explicaron los objetivos del estudio y firmaron un consentimiento informado. Después, completaron el cuestionario IADA (se obtuvo autorización de uso para la investigación a las autoras), con un tiempo aproximado de 30 minutos. Se usó el software spss versión 21 para los análisis de datos, los cuales incluyeron un análisis bivariado descriptivo por sexo entre las puntuaciones de las dimensiones del IADA.

Se hicieron pruebas de hipótesis, previa verificación del supuesto de normalidad, el cual se efectuó mediante el contraste de Kolmogorov-Smirnov. Se utilizó la prueba t de Student en el análisis de la prevalencia de vulnerabilidad a los riesgos por sexo entre la muestra, y la prueba chi-cuadrado $\left(\mathrm{X}^{2}\right)$ entre los grupos comparados. Como análisis complementario, se utilizaron las pruebas de Hedges ajustada $\left(\mathrm{g}_{\text {(ajustada) }}\right)$ y de Cramer (V) como estimadores del tamaño del efecto existente. Dicha presencia fue estimada en puntuaciones iguales o superiores a 0,2 para efectos pequeños, a 0,5 para efectos moderados y a 0,8 para efectos grandes (Cohen, 1992).

En lo relativo a la verificación de la fiabilidad, se utilizó el coeficiente de consistencia interna alfa de Cronbach ( $\alpha$ ). De acuerdo con Vallejo (2007), las puntuaciones de fiabilidad entre 0,60 y 0,80 son consideradas aptas, aunque puntuaciones sobre 0,50 también son consideradas válidas (Nunnally, 1978). Finalmente, se hicieron análisis sobre estimaciones de riesgo, u odds ratio (or), de variables sociodemográficas en las situaciones y las conductas de riesgo de los adolescentes para conocer sus estimaciones relevantes. Un or se considera un factor de riesgo cuando se da la presencia de la unidad (1) entre los intervalos de confianza al 95\%, ya que así se consideraría como estadísticamente significativo (Cerda, Vera y 
Rada, 2013; Pardo y Ruiz, 2010). Se utiliza este procedimiento estadístico porque el objetivo en este punto consiste en establecer factores de riesgo asociados a la población adolescente.

\section{Diseño}

El estudio corresponde a uno descriptivo, comparado por sexo, de los niveles de práctica de riesgos en adolescentes participantes. Así mismo, se analizan las proporciones de niveles elevados de riesgo para identificar la presencia de grupos vulnerables, y se analizan factores de riesgo asociados a situaciones y conductas de riesgo entre los adolescentes.

\section{Resultados}

\section{Verificación de normalidad, medias globales por áreas y análisis de consistencia interna}

Se cumple el supuesto de normalidad ( $p>0,05)$. Los análisis descriptivos por género muestran en la dimensión Familia puntajes de $M_{\text {hombres }}=82,9$ puntos; $D T=43,9$ y $M_{\text {mujeres }}=92,1$ puntos; $D T=43,6$. En la dimensión Social, los puntajes fueron de $M_{\text {hombres }}=39,1$ puntos; $D T=12,5$ y $M_{\text {mujeres }}=36,4$ puntos; $D T=9,4$. En la dimensión Escolar, los puntajes alcanzados fueron $M_{\text {hombres }}=49,5$ puntos; $D T=25,2$ y $M_{\text {muieres }}=54,4$ puntos; $D T=23,4$. En la dimensión Personal, se obtuvieron puntajes de $M_{\text {hombres }}=51,4$ puntos; $D T=34,5$ y $M_{\text {muieres }}=57,6$ puntos; $D T=32,2$. En la dimensión Salud, los puntajes fueron de $M_{\text {hombres }}=53,7$ puntos; $D T=42,7$ y $M_{\text {mujeres }}=37,1$ puntos; $D T=23,9$. Los puntajes globales alcanzados muestran resultados de
$M_{\text {hombres }}=286,5$ puntos; $D T=125,5$ y $M_{\text {mujeres }}=281,1$ puntos; $D T=92,9$. Se hallaron diferencias estadísticamente significativas únicamente en la dimensión Salud, en la que los hombres puntuaron mayor riesgo que las mujeres $t(300)=3,094, p=0,003$.

Los efectos entre los grupos analizados muestran efectos pequeños $\left(g_{(\text {ajustada) }}>0,2\right)$ en las dimensiones Familia $\left(g_{(\text {ajustada) }}=-0,21\right)$, Social $\left(g_{\text {(ajustada) }}=0,21\right)$, Escolar $\left(g_{(\text {(ajustada) }}=-0,2\right)$ y Salud $\left(g_{\text {(ajustada) }}=0,42\right)$. Se puede apreciar que las mujeres puntúan ligeramente más en las dimensiones Familia y Escolar, mientras que los hombres lo hacen en las dimensiones Social y Salud.

La consistencia interna global de la escala IADA fue de $\alpha=0,93$. Por áreas, se observó que todas presentaron una buena consistencia interna $(\alpha>0,70)$, a excepción del área social $(\alpha=0,50)$, por lo que las estimaciones de la prueba se consideran moderadamente altas con la respectiva excepción. Todas las medias de las puntuaciones y la consistencia interna se reportan en la tabla 1.

Para conocer la proporción de participantes que cuentan con criterios de riesgo entre sus dimensiones y el puntaje global, se clasificaron los puntajes que estén por encima de la puntuación $T_{(80)}$ en cada uno de los casos. Del análisis se desprende que en la dimensión Familia el 11,2\% en el segmento de hombres y el 17,4\% en el grupo de mujeres cuentan con criterios de riesgo. En la dimensión Social, la tasa de riesgo en los hombres es de $11,6 \%$ y en las mujeres es de $8,7 \%$. Mientras que en la dimensión Escolar la tasa es de 21,9\% en los hombres y de 20,3\% en las mujeres. Además, en la dimensión Personal se encontraron prevalencias de 8,6\% en los hombres y de $11,6 \%$ en las mujeres. En la dimensión Salud, la tasa fue del $8,2 \%$ y no hubo frecuencias en las mujeres. En la evaluación global del IADA, el 8,6\% de los

Tabla 1

Análisis comparado por sexo y consistencia interna del IADA adolescentes de bachillerato

\begin{tabular}{|c|c|c|c|c|c|c|}
\hline \multirow{2}{*}{ Dimensión } & Hombres & Mujeres & \multirow{2}{*}{ Dif. ${ }_{[\text {IC 95\%] }}$} & \multirow{2}{*}{$\mathrm{t}_{(300)} ; \mathrm{p}$} & \multirow{2}{*}{$\mathrm{g}_{\text {(ajust.) }}$} & \multirow{2}{*}{ Alfa } \\
\hline & M (Ds) & M (Ds) & & & & \\
\hline Familia & $82,91(43,96)$ & $92,04(43,6)$ & $9,13_{[-2,71-20,97]}$ & $-0,492 ; 0,13$ & $-0,21$ & 0,74 \\
\hline Social & $39,01(12,57)$ & $36,49(9,49)$ & $-2,52_{[-5,74-, 7]}$ & 1,$540 ; 0,125$ & 0,21 & 0,50 \\
\hline Escolar & $49,52(25,26)$ & $54,42(23,43)$ & $4,9_{[-1,8-11,61]}$ & $-1,438 ; 0,151$ & $-0,20$ & 0,71 \\
\hline Personal & $51,46(34,53)$ & $57,67(32,23)$ & $6,28_{[-2,87-15,43]}$ & $-1,331 ; 0,178$ & $-0,18$ & 0,83 \\
\hline Salud & $53,71(42,72)$ & $37,06(23,95)$ & $-15,78_{[-26,26--5,31]}$ & 3,$094 ; 0,003$ & 0,42 & 0,84 \\
\hline Global & $286,55(125,57)$ & $281,12(92,97)$ & $2,01_{[-31,57-35,59]}$ & 0,$333 ; 0,906$ & 0,05 & 0,93 \\
\hline
\end{tabular}

Nota. 302 observaciones. Elaboración propia 
hombres y el 2,9\% de las mujeres muestran riesgo generalizado. No hubo diferencias estadísticamente significativas $(p<0,05)$ entre los resultados comparados por sexo de cada una de las dimensiones, y en ninguno de los casos se encontraron tamaños del efecto representativos $(V>0,2)$ entre los grupos comparados (ver tabla 2).

Se destaca la presencia significativa de prevalencias de riesgo superiores al $20 \%$ tanto en hombres como en mujeres adolescentes en la dimensión Escolar; mientras que en el caso de las mujeres destacan los riesgos relacionados con la dimensión Familia que bordea el 17\% aproximadamente, lo que evidencia un sector del análisis que presenta criterios de vulnerabilidad con respecto a la muestra analizada.

\section{Estimaciones de riesgo y factores de riesgo en los adolescentes}

Tabla 2

Prevalencia de riesgo comparado por género

\begin{tabular}{|c|c|c|c|c|c|c|c|c|}
\hline \multirow{2}{*}{ Dimensión } & \multicolumn{2}{|c|}{ Hombres; $\mathbf{n}=233$} & \multicolumn{2}{|c|}{ Mujeres; $n=69$} & \multirow{2}{*}{$\mathrm{X}^{2} ; \mathrm{p}$} & \multirow{2}{*}{$\mathrm{V}$} & \multicolumn{2}{|c|}{ General } \\
\hline & Frec. & $\%$ & Frec. & $\%$ & & & Frec. & $\%$ \\
\hline Familia & 26 & $11,2 \%$ & 12 & $17,4 \%$ & 1,$88 ; 0,124$ & 0,08 & 38 & $12,6 \%$ \\
\hline Social & 27 & $11,6 \%$ & 6 & $8,7 \%$ & 0,$46 ; 0,334$ & 0,04 & 33 & $10,9 \%$ \\
\hline Escolar & 51 & $21,9 \%$ & 14 & $20,3 \%$ & 0,$08 ; 0,46$ & 0,02 & 65 & $21,5 \%$ \\
\hline Personal & 20 & $8,6 \%$ & 8 & $11,6 \%$ & 0,$57 ; 0,293$ & 0,04 & 28 & $9,3 \%$ \\
\hline Salud & 19 & $8,2 \%$ & 0 & $0 \%$ & 6; 0,006 & 0,14 & 19 & $6,3 \%$ \\
\hline Global & 20 & $8,6 \%$ & 2 & $2,9 \%$ & 2,$55 ; 0,084$ & 0,09 & 22 & $7,3 \%$ \\
\hline
\end{tabular}

Nota. 302 observaciones. Elaboración propia

Se consideró conocer si variables como el sexo (ser mujer), la edad (tener 15 años o menos), el centro educativo de estudios (institución privada-religiosa), la ubicación domiciliaria (sector urbano), la identificación étnica (no ser mestizo), la presencia de hermanos (ser hijo único) y los antecedentes psicológicos individuales o familiares (tener antecedentes) constituyen una estimación de riesgo o un factor de riesgo en las puntuaciones riesgosas de los adolescentes en las áreas de análisis del IADA.

Del presente análisis, se destaca en la dimensión Familia que el grupo de personas que cuentan con antecedentes psicológicos individuales y familiares están más expuestos al riesgo 3,2 veces y 3,3 veces que el grupo sin antecedentes. En la dimensión Social, el grupo de estudiantes menores de 15 años están 4,5 veces más expuestos al riesgo que el resto y 2,7 veces más expuestos los que cursan sus estudios en una institución privada-religiosa.

En la dimensión Escolar, los adolescentes con antecedentes psicológicos individuales y familiares están 2,3 veces y 3,3 veces más expuestos que el resto de participantes. En la dimensión Personal, los estudiantes menores de 15 años están expuestos 3,7 veces más y aquellos con antecedentes psicológicos familiares lo están 2,4 veces más que los otros grupos.

En la dimensión Salud, el grupo de adolescentes que estudian en una institución privada-religiosa tienen 15,7 veces mayor exposición que el resto, y aquellos que presentan antecedentes psicológicos individuales y familiares tienen 2,9 veces mayor exposición. Además, en la evaluación global del riesgo están expuestos seis veces más aquellos menores de 15 años; 8,8 veces más quienes estudian en una institución privada-religiosa; 4,9 veces más quienes cuentan con antecedentes psicológicos individuales y 5,5 veces más los que tienen antecedentes psicológicos familiares (ver tabla 3).

En cuanto a cuáles indicadores comprenden ser factores de riesgo, se encontró que el grupo cuyo criterio relacionado con el sexo coincide con el ser mujer comprende un factor de riesgo en la dimensión Salud OR $=1,3 ; 95 \%$ CI $[1,2-1,4] ; p<0,05$, por lo que se considera como significativo. También, la edad relacionada con tener 15 años o menos representa un factor de riesgo en las dimensiones Social $O R=4,5 ; 95 \% \mathrm{CI}$ $[1,1-19,5], p<0,05$ y Salud $O R=1,3 ; 95 \%$ CI $[1,2-1,4]$, $p<0,05$. La condición de no autoidentificarse con la 
Tabla 3

Estimaciones de riesgo y factores de riesgo de los adolescentes

\begin{tabular}{|c|c|c|c|c|c|c|}
\hline \multirow{2}{*}{ Estimaciones de riesgo } & Familia & Social & Escolar & Personal & Salud & IADA \\
\hline & $\mathbf{O R}_{[\mathrm{IC} 95 \%]}$ & $\mathbf{O R}_{[\mathrm{IC} 95 \%]}$ & $\mathbf{O R}_{[\mathrm{IC95 \% ]}}$ & $\mathbf{O R}_{[\mathrm{IC95 \% ]}}$ & OR $_{[\mathrm{IC} 95 \%]}$ & OR $_{[\mathrm{IC} 95 \%]}$ \\
\hline Ser mujer & $1,7_{[0,8-3,5]}$ & $0,73_{[0,3-1,8]}$ & $0,91_{[0,5-1,8]}$ & $1,4_{[0,6-3,3]}$ & $1,3_{[1,2-1,4]}^{*}$ & $0,3_{[0,1-1,4]}$ \\
\hline Menor de 15 años & $1,2_{[0,5-2,9]}$ & $4,5_{[1,1-19,5]}^{*}$ & $0,95_{[0,5-1,9]}$ & $3,7_{[0,9-16,1]}$ & $1,3_{[1,2-1,4]}^{*}$ & $6_{[0,8-45,3]}$ \\
\hline No ser mestizo & $1_{[0,4-2,6]}$ & $0,97_{[0,4-2,6]}$ & $0,84_{[0,4-1,8]}$ & $2_{[0,78-4,8]}$ & $1,5_{[0,5-4,7]}$ & $1,2_{[0,4-3,8]}$ \\
\hline Ser hijo único & $1_{[0,3-3,7]}$ & $0,4_{[0,1-2,7]}$ & $0,53_{[0,2-1,8]}$ & $1,5_{[0,4-5,5]}$ & $0,7_{[0,1-5,1]}$ & $0,6_{[0,2-1,5]}$ \\
\hline Entorno urbano & $1,2_{[0,6-2,6]}$ & $1,3_{[0,6-3]}$ & $1,2_{[0,7-2,2]}$ & $0,53_{[0,2-1,2]}$ & $1,1_{[0,4-2,9]}$ & $0,8_{[0,3-2,1]}$ \\
\hline Inst. privada-religiosa & $1,6_{[0,8-3,3]}$ & $2,7_{[1,2-6,2]^{*}}$ & $1,5_{[0,8-2,6]}$ & $1,1_{[0,5-2,3]}$ & $15,7_{[2-19]^{*}}$ & $8,88_{[2-38,3]}^{*}$ \\
\hline Ant. individuales & $3,2_{[1,6-6,5]}^{*}$ & $2_{[0,9-4,4]}$ & $2,3_{[1,3-4,3]} *$ & $1,8_{[0,8-4,3]}$ & $2,9_{[1,1-0,5]}^{*}$ & $4,9_{[1,7-0,2]}$ * \\
\hline Ant. familiares & $3,3_{[1,6-0,8]}^{*}$ & $1,9_{[0,8-4,3]}$ & $3,3_{[1,7-6,2]}$ * & $2,4_{[1-5,7]}$ & $2,9_{[1,1-7,8]} *$ & $5,5_{[2,2-13,5]}$ * \\
\hline
\end{tabular}

Nota. ${ }^{*}$ Factor de riesgo. oR $=$ odds ratio. Elaboración propia

etnia mestiza, el hecho de ser hijo único y el hecho de tener domicilio dentro de la zona urbana no mostraron ningún elemento asociado como factor de riesgo. Por otra parte, estudiar en una institución privada-religiosa mostró que comprende un factor de riesgo en las dimensiones Social oR $=2,7 ; 95 \% \mathrm{CI}$ $[1,2-6,2], p<0,05$, Salud oR $=15,7 ; 95 \%$ CI [2-119], $p<0,05$ y en la evaluación global del IADA $O R=8,8$; $95 \%$ CI $[2-38,3], p<0,05$. Los antecedentes psicológicos individuales es un factor de riesgo asociado a los riesgos en las dimensiones Familia $O R=3,2 ; 95 \% \mathrm{CI}$ $[1,6-6,5], p<0,05$, Escolar oR $=2,3 ; 95 \%$ CI $[1,3-4,3]$, $p<0,05$, Salud $O R=2,9 ; 95 \%$ CI $[1.1-7,5], p<0,05$ y en la evaluación global de riesgo (IADA) $O R=4,9 ; 95 \% \mathrm{CI}$ [1,7-10,2], $p<0,05$. Así mismo, los antecedentes psicológicos familiares representan un factor de riesgo en las dimensiones Familia OR $=3,3 ; 95 \%$ CI $[1,6-6,8]$, $p<0,05$, Escolar oR $=3,3 ; 95 \%$ CI $[1,7-6,2], p<0,05$, Salud $O R=2,9 ; 95 \%$ CI $[1,1-7,8], p<0,05$ y en la evaluación global del IADA $O R=5,5 ; 95 \%$ CI $[2,2-13,5]$, $p<0,05$.

\section{Discusión}

El objetivo del presente estudio es identificar los perfiles de riesgo psicosocial en adolescentes escolarizados de Ambato, dado que no se encuentran otros estudios en Ecuador que describan estos perfiles; también, conocer la fiabilidad de la prueba a través de la consistencia interna entre los ítems.

En primer lugar, en cuanto a la consistencia interna de las puntuaciones, se encontró que estas fluctúan entre 0,71 (área escolar) y 0,84 (área salud), con excepción del área social con un puntaje de 0,5 ; el puntaje global muestra una consistencia interna de $a=0,93$. Estos resultados tienen semejanza con los criterios psicométricos de la versión de validación de la prueba de Gómez-Maqueo, Barcelata y Durán (2010), aplicada en población adolescente mexicana. Estos datos apuntan a considerar que la prueba es moderadamente fiable y con evidencia de su aplicabilidad en población adolescente ecuatoriana, por lo que coinciden con los criterios tanto de Nunnally (1978) como de Vallejo (2007), esto desde luego con fines investigativos. La aplicabilidad de la prueba para la realización de diagnósticos individuales debe ser tomada con precaución, ya que una de las áreas (social) muestra una fiabilidad media.

Se identifica que los hombres muestran una presencia marcada de conductas y situaciones de riesgo en las dimensiones Social y Salud, en tanto que en las mujeres se presentan en las dimensiones de Familia, Escolar y Personal. Desde un análisis global del IADA, los estudiantes varones puntúan mucho más que las mujeres, hallazgo que coincide con los encontrados en otros estudios (Barcelata, Durán y Lucio, 2004; Campo-Arias et al., 2008). De este modo, la condición de ser hombre incide en el incremento del riesgo, lo que podría entenderse desde los mecanismos culturales de socialización y de crianza de los adolescentes (Herrera, 1999; Huitrón-Bravo et al., 2011).

Respecto a la vulnerabilidad, se encontraron subgrupos con criterios de riesgo elevado en la dimensión Escolar, siendo mayor en hombres (20\%) que en mujeres (17\%) aspectos que se asocian a conductas problemáticas, repetición del año escolar, dificultades en el 
aprendizaje o indiferencia. Tal como lo sugiere Maria Angela Mattar Yunes (2001), la vulnerabilidad en este nivel puede ser asociada a las limitadas oportunidades o a las dificultades internas o externas que incrementan los riesgos. Por otra parte, en la dimensión Familia, la vulnerabilidad está presente en el $17 \%$ en mujeres, lo cual incluye problemas emocionales, conflictos con el sistema parental y desaprobación social. Estos hallazgos se contraponen a lo señalado por Campo-Arias et al. (2008), quienes consideran que en los varones suele haber mayor prevalencia de conductas de riesgo.

En las estimaciones de riesgo, se destaca que los participantes menores de 15 años presentan mayor exposición a los riesgos en la dimensión Social y a los riesgos integrales del adolescente. La explicación de estos resultados se podría relacionar con los aspectos amenazantes a los que se pueden enfrentar los jóvenes y con que a una menor edad se dificulta más la capacidad para asumirlos (Coleman, Hendry y Kloeb, 2007). Así mismo, la pertenencia a una institución de carácter privado-religioso promueve una mayor exposición a los riesgos relacionados con la salud y a los riesgos generales del adolescente. Estos datos no son concordantes con lo señalado por Cardozo y Alderete (2009) sobre las condiciones socioeconómicas precarias en la desadaptación y los síntomas psicológicos, sino que revelan que una condición económica media-alta en esta muestra incrementa las exposiciones y los riesgos.

En cuanto a los adolescentes con antecedentes psicológicos individuales o familiares, están expuestos a mayores riesgos integrales que quienes no los tienen. Estos resultados concuerdan con estudios previos (Haquin, Larraguibel y Cabezas, 2004) que reportan indicadores de riesgo en adolescentes con indicadores psicopatológicos. De tal manera, estos resultados dan indicios de la comprensión de la relación entre comportamientos y situaciones de riesgo en adolescentes, las condiciones y las áreas en que pueden originarse signos de psicopatología juvenil o la iniciación de conductas delictivas. Esto plantea desafíos acerca del abordaje que se necesita llevar a cabo en planes de prevención e intervención (Offord y Kraemer, 2000).

\section{Conclusiones}

Aquí, es posible mencionar al menos tres consideraciones. En primer lugar, los planes preventivos deben orientarse hacia diferentes áreas del adolescente, especialmente hacia la salud. En segundo lugar, los comportamientos de riesgo están interconectados y no constituyen conductas aisladas, se requiere intervenir en "hábitos o estilos de vida" de riesgo, lo que redunda en acciones a corto, a mediano y a largo plazo, de tal forma que una campaña de salud en educación sexual debe abordar las relaciones familiares o las relaciones sociales con pares. En tercer lugar, se requiere un plan de prevención e intervención ante comportamientos de riesgo que distinga el sexo en adolescentes. Como se ha observado, hay exposiciones al riesgo específicas en adolescentes hombres $y$ en mujeres, que requieren ser tomadas en cuenta en campañas de prevención o intervención. En conjunto, estas tres concepciones podrían disminuir la exposición y la frecuencia de la práctica de comportamientos de riesgo de los adolescentes en Ambato.

\section{Limitaciones y futuros estudios}

Se debe considerar que en el estudio hay una elevada presencia de participantes del sexo masculino, así como de sujetos procedentes de una institución educativa de carácter religioso-privado, lo cual incide en la representatividad de los adolescentes en la ciudad de Ambato. Una segunda limitación se refiere a la baja consistencia interna que se observó en algunas áreas, específicamente la social, que pudo afectar la objetividad de la prueba y la ausencia de resultados en esta misma área; de allí que estos resultados deban ser tomados con reserva. Al respecto, futuros estudios requieren la adopción de medidas para asegurar la representatividad de los adolescentes que estudian en Ambato; en concreto, incluir mayor población femenina y adolescentes de establecimientos públicos. Por otro lado, sobre la capacidad evaluativa del IADA, se requiere examinar las propiedades psicométricas del instrumento en población ecuatoriana y explorar la estructuración factorial de los ítems (Fernández, 2004), así como explorar criterios de validez discriminante, convergencia y estabilidad. Complementario a esto, sería de interés incorporar otras variables sociodemográficas que se relacionan con los comportamientos de riesgo (e. g., conducta sexual, conductas alimentarias o preocupación por el cuerpo y adherencia a pandillas, entre otras).

El presente trabajo resulta pionero en incorporar las complejidades de la diversidad de los comportamientos de riesgo en adolescentes escolarizados en Ecuador. Esto alienta a explorar otros aspectos en diversas zonas del país que faciliten la inclusión de estos 
comportamientos en los planes de prevención y/o intervención con adolescentes.

\section{Referencias}

Arce, L. (1995). Conflictos en la evolución del adolescente. Teoría y metodología para la intervención en familias. San José: Universidad Nacional de Costa Rica.

Arnett, J. (1992). Reckless behaviour in adolescence: A developmental perspective. Developmental Review, 12, 339-373. doi: 10.1016/0273-2297(92)90013-R

Arnett, J. (1999). Adolescent storm and stress, reconsidered. American Psychologist, 54(6), 317-326. doi: 10.1037/0003-066X.54.5.317

Barcelata, B., Durán, C. y Lucio, E. (2004). Indicadores de malestar psicológico en un grupo de adolescentes mexicanos. Revista Colombiana de Psicología, (13), 64-73.

Bardagi, M., Arteche, A. y Neiva-Silva, L. (2005). Projetos sociais com adolescentes em situação de risco: discutindo o trabalho e a orientação profissional como estratégias de intervenção. En C. Hutz (Org.), Violência e risco na infância e na adolescência: pesquisa e intervenção (pp. 101-146). São Paulo: Casa do Psicólogo.

Becoña, E. (2002). Bases científicas de la prevención de las drogodependencias. Madrid: Delegación del Gobierno para el Plan Nacional de Drogas.

Bozhovich, L. (1976). La personalidad y su formación en la edad infantil. La Habana. La Habana: Pueblo y Educación.

Campo-Arias, A., Cogollo, Z. y Díaz, C. (2008). Comportamientos de riesgo para la salud en adolescentes estudiantes: prevalencia y factores asociados. Salud Uninorte, 24, 226-234. doi: 10.1590/S0104-11692010000200005

Cardona, O. (2001). La necesidad de repensar de manera holística los conceptos de vulnerabilidad y riesgo. Una crítica y una revisión necesaria para la gestión. Ponencia presentada en International Work-Conference on Vulnerability in Disaster, Theory and Practice. Wageningen, Holanda.

Cardozo, G. y Alderete, A. M. (2009). Adolescentes en riesgo psicosocial y resiliencia. Psicología desde el Caribe. Universidad del Norte, (23), 148-182.

Comisión Económica para América Latina y el Caribe. (Cepal). (2001). Informe de la Reunión de Expertos: Seminario Internacional sobre las Diferentes Expresiones de la Vulnerabilidad Social en América Latina y el Caribe. Santiago de Chile: División de Población de la Cepal/Celade.

Cerda, J., Vera, C. y Rada, G. (2013). Odds ratio: Aspectos teóricos y prácticos. Revista Médica de Chile, 141, 1329-1335. doi: 10.4067/S0034-98872013001000014
Cohen, J. (1992). A power primer. Psychological Bulletin, 112(1), 155-159. doi: 10.1037/0033-2909.112.1.155

Coleman, J., Hendry, L. y Kloeb, M. (2007). Adolescence and health. West Sussex: John Wiley \& Sons.

Fergusson, D., Woodward, L. y Horwood, L. (2000). Risk factors and life processes associated with the onset of suicidal behaviour during adolescence and early adulthood. Psychological Medicine, 30, 23-39. doi: 10.1017/ S003329179900135X

Fernández, T. (2004). Multicolinealidad: guía de clase. México D. F.: El Colegio de México-Centro de Estudios Sociológicos.

Fisher, S.y Smith, G. T. (2008). Binge eating, problem drinking, and pathological gambling: Linking behavior to shared traits and social learning. Personality and Individual Differences, 44, 789-800. doi: 10.1016/j.paid.2007.10.008

Friedman, R. (2006). Uncovering an epidemic-screening for mental illness in teens. New England Journal of Medicine, 355, 2717-2719. doi: 10.1056/NEJMp068262

Gómez-Maqueo, Barcelata, B. y Durán, C. (2010). Inventario Autodescriptivo del Adolescente (IADA). Manual de aplicación. México D. F.: Editorial Manual Moderno.

Haquin, C., Larraguibel, M. y Cabezas, J. (2004). Factores protectores y de riesgo en salud mental en niños y adolescentes de la ciudad de Calama. Revista Chilena de Pediatría, 75(5), 425-433. doi: 10.4067/S037041062004000500003

Herrera, P. (1999). Principales factores de riesgo psicológicos y sociales en el adolescente. Revista Cubana de Pediatria, 71(1), 39-42.

Huitrón-Bravo, G., Denova-Gutiérrez, E., Halley-Castillo, E., Santander-Rigollet, S., Bórquez-Puga, M., Zapata, P. L., et al. (2011). Conductas de riesgo en una muestra de adolescentes chilenos y mexicanos: un estudio comparativo. Papeles de Población, 70, 33-47.

International Federation of Red Cross (IFRC). (2014, agosto 10). ¿Qué es la vulnerabilidad? Recuperado de http://www.ifrc. org/es/introduccion/disaster-management/sobre-desastres/que-es-un-desastre/que-es-la-vulnerabilidad/

Instituto Nacional de Estadística y Censos (INEC). (2010). Censo de Población y Vivienda. Quito: INEC.

Irwin, C. E. (1990). The theoretical concept of risk adolescent. Adolescent Medicine: State of the Art Reviews, 1, 1-14. doi: 10.1007/978-1-4614-6633-8_2

Larson, R. (1988). The high school "Junior Theme" as an adolescent rite of passage. Journal of Youth and Adolescence, 17(4), 267-283. doi: 10.1007/BF01537670 
Mattar Yunes, M. A. (2001). A questão triplamente controvertida da resiliencia em famílias de baixa renda. São Paulo: Pontifícia Universidade Católica de São Paulo.

Ministerio de Educación de Ecuador (2014a). Indicadores educativos de asistencia por edad. Recuperado de https:// goo.gl/drC7E6

Ministerio de Educación de Ecuador (2014b). Tasa bruta de asistencia al bachillerato. Recuperado de https://goo. $\mathrm{gl} / \mathrm{gg} 2260$

Ministerio de Educación de Ecuador (2014c). Tasa neta de asistencia al bachillerato. Recuperado de https://goo. $\mathrm{gl} / \mathrm{zGRwFx}$

Nunnally, J. (1978). Psychometric theory. Nueva York: McGraw-Hill.

Offord, D. y Kraemer, H. (2000). Risk factors and prevention. EвMH Notebook, (3), 70-71. doi: 10.1136/ebmh.3.3.70

Palacios, J. y Andrade, P. (2007). Desempeño académico y conductas de riesgo en adolescentes. Revista de Educación y Desarrollo, 7, 5-16.

Páramo, M. D. (2011). Factores de riesgo y factores de protección en la adolescencia: análisis de contenido a través de grupos de discusión. Terapia Psicológica, 29(1), 85-95. doi: 10.4067/S0718-48082011000100009

Pardo, A. y Ruiz, M. (2010). Análisis de datos en Ciencias Sociales y de la Salud II. Madrid: Síntesis.

Rutter, M. (1987). Psychosocial resilience and protective mechanism. American Journal of Orthopsychiatry, 57(3), 316-331. doi: 10.1111/j.1939-0025.1987.tb03541.x
Sánchez-González, D. y Egea-Jiménez, C. (2011). Enfoque de vulnerabilidad social para investigar las desventajas socioambientales. Su aplicación en el estudio de los adultos mayores. Papeles de Población, 69, 151-185.

Sinha, J. W. (2007). Adolescent risk behaviors and religion: Findings from a national study. Journal of Adolescent, 30, 231-249. doi: 10.1016/j.adolescence.2006.02.005

Toro-Trallero, J. (2010). El adolescente en su mundo. Riesgos, problemas y trastornos. Madrid: Pirámide.

United Nations International Strategy for Disaster Reduction (UNISDR). (2009). Terminología sobre reducción de riesgo de desastres. Ginebra: UNISDR. Recuperado de http:// www.unisdr.org/files/7817_UNISDRTerminologySpanish.pdf

Vallejo, P. (2007). Estadística aplicada a las Ciencias Sociales: la fiabilidad de los test y escalas. Madrid: Universidad Pontificia Comillas.

Vargas-Trujillo, E., Henao, J. y González, C. (2007). Toma de decisiones sexuales y reproductivas en la adolescencia. Acta Colombiana de Psicología, 10, 49-63.

Villar-Torres, P., Luengo-Martín, M. A., Gómez-Fraguela, X. A. y Romero-Triñanes, E. (2003). Una propuesta de evaluación de variables familiares en la prevención dela conducta problema en la adolescencia. Psicothema, 15, 581-588.

Witkiewitz, K. y Marlatt, A. (2004). Relapse prevention for alcohol and drugs problems. American Psychology, 59(4), 224-235. doi: 10.1037/0003-066X.59.4.224 